\title{
Orbit Design for Thai Space Consortium Satellite ${ }^{\dagger}$
}

\author{
Suwat Sreesawet ${ }^{*}$, Seksan Jaturat * and Sittiporn Channamsin *(D) \\ Geo-Informatics and Space Technology Development Agency (Public Organization), 88, Moo 9, \\ Tambol Thung Sukhla, Si Racha District 20230, Chon Buri Province, Thailand \\ * Correspondence: suwat@gistda.or.th (S.S.); seksan@gistda.or.th (S.J.); sittiporn@gistda.or.th (S.C.); \\ Tel.: +66-033-046-315 (S.S. \& S.J. \& S.C.) \\ + Presented at the Innovation Aviation \& Aerospace Industry-International Conference 2020 (IAAI 2020), \\ Chumphon, Thailand, 13-17 January 2020.
}

Published: 27 December 2019

check for updates

\begin{abstract}
Currently, Geo-Informatics and Space Technology Development Agency (GISTDA), National Astronomical Research Institute of Thailand (NARIT) and Synchrotron Light Research Institute (SLRI) have a co-operation on a project of developing a satellite for scientific research called Thai Space Consortium (TSC). The project is aiming at Earth remote sensing mission by a small satellite about $100 \mathrm{~kg}$. The main payload of the satellite is an optical instrument with the secondary payload of energetic particle detector for space weather. The satellite is designed to be in a Sun Synchronous orbit due to requirement of same light condition throughout the operational lifetime. In the meantime, there is another project by GISTDA named THEOS-2. This project consists of two remote-sensing satellites, THEOS-2 MainSAT and THEOS-2 SmallSAT, under the development in Europe. The SmallSAT does not have the propulsion subsystem. So it cannot perform station-keeping maneuvers or maintain constellation with others. Therefore, in this paper, we analyze two scenarios that the TSC satellite flies as constellations with the MainSAT of THEOS2 project. The constellation is in the sense that the TSC satellite flies on the same ground track path with the MainSAT satellite with slightly differences in local solar time. The ground track sequencing is presented with a methodology for obtaining orbital parameters with a discussion on accuracy relating to Keplerian assumption.
\end{abstract}

Keywords: mission analysis; sun synchronous orbit

\section{Introduction}

Thai Space Consortium (TSC) is originally a co-operation project of Geo-Informatics and Space Technology Development Agency (GISTDA), National Astronomical Research Institude of Thailand (NARIT) and Synchrotron Light Research Institute (SLRI). The main activity under TSC is to develop a Thai small satellite for earth-remote sensing mission. The satellite will have the primary payload of an optical instrument and the secondary payload of energetic particle detector. Meanwhile, there are an ongoing space project by GISTDA named THEOS-2. One part of the project is the deployment of two satellites, THEOS-2 MainSAT and THEOS-2 SmallSAT. The main payloads of both satellites of THEOS-2 program are optical Earth observing camera. The MainSAT has a propulsion subsystem meaning that it is capable of performing station-keeping maneuver. On the other hand, the SmallSAT will not have the propulsion and could not maintain its orbit. So, in this paper, we design two satellite orbits that are a constellation with the MainSAT of THEOS-2 program.

The MainSAT and SmallSAT will be placed in sun-synchonous orbits (SSO) which are one of the most commonly used orbits for Earth observation mission, for example WorldView 1-4 from DigitalGlobe ${ }^{\circledR}$ and Landsat 1-9 from NASA. The main reason of popularity is the lighting conditions of ground track which remain constant throughout a mission lifetime. The mission design procedure 
for this orbit has been well established in Ref. [1-4]. Some studies use an assumption of unperturbed two-body motion in the design method, i.e., Keplerian motion [1,3].

In this paper, we present an iterative methodology for designing orbits which takes perturbations into an account. The reference orbit of THEOS-2 MainSAT is described with the methodology used in the paper. Then, the designed orbits are presented with a discussion of the inaccuracy of Keplerian assumption.

\section{Reference Orbit and Design Methodology}

The goal of the this study is to design the orbit of the Thai space consortium small to fly as a constellation with the THEOS2 MainSAT. MainSAT, which will be launched in 2021, will be in a Sun Synchronous orbit with 26 days of repetitive ground track. The satellite will complete 385 revolutions before coming back to the initial location respected to the earth surface. So it will have the mean motion of $14+21 / 26$ revolutions per day. By the larger number of revolutions before repeating ground track, the satellite has a denser traveling path around the Earth. The example of MainSAT ground track's density is shown in Figure 1 by assuming that the first ascending node is at $0^{\circ}$ longitude.

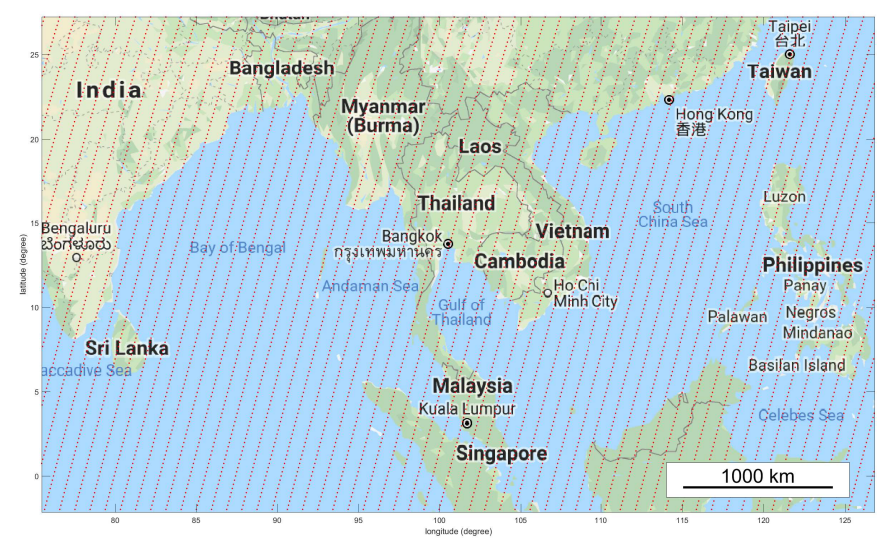

Figure 1. The estimated descending ground track of THEOS2 main satellite.

The preliminary orbit of THEOS2 is specified in the Table 1 . The given orbital elements in the Table are osculating orbit parameters expressed on $\mathrm{J} 2000$ inertial reference frame.

\subsection{Design of Revisiting Time}

The Low-Earth orbit is defined by the altitude of $200 \mathrm{~km}$ and up to $1000 \mathrm{~km}$. The upper limit of thousand kilometers is based on the lowest height before exposing to the hazardous region of Van Allen radiation belt. By limitation of altitude, the number of flight revolutions during $86,400 \mathrm{~s}$, a day, stays in between 13 to 16 revolutions [1]. If the revolutions during a day is a whole number, satellite repeats the same ground track everyday. Even the highest whole number of 16 revolutions, the adjacent ground track would be $2504.69 \mathrm{~km}$ at the equator which is too coarse for such a mission. In order to achieve finer ground tracks, mission designers can select the number of revolutions to be a rational number between 13-16. The finer grids, however, lead to the longer revisiting time. For example THEOS1 flies at $14+5 / 26$ revolutions per days which means that it flies 369 revolutions in 26 days and repeats the same ground track. We also can write it as " $26 D 369 R$ ". Then, we can form a simple relationship as following

$$
N=\frac{R}{D}
$$


where $R \in \mathbb{N}$ and $D \in \mathbb{N}$ are whole numbers of revolution and days for repeating ground track and $N$ is mean motion in the unit of revolutions per day. Also, from a simple geometrical relationship, we can find the distance at the equator between adjacent node, $G$, which is

$$
G=\frac{2 \pi}{R} R_{E}
$$

where $R_{E}$ is Earth's radius at equator which is $6378.137 \mathrm{~km}$ according to WGS84 standard. Once we know the number of orbits per days, we can find nodal period, $P$, by the following relationship

$$
P=\frac{86400}{N} \text {. }
$$

Later, we will use the nodal period in seconds, $P$, for determining the orbital parameters of the designed orbit.

Table 1. Refercence orbit of THEOS2 main satellite.

\begin{tabular}{cc}
\hline Epoch Time & 1 January 2021 22:00:00.000 \\
\hline semi-major axis $(a)$ & $7008.4715082(\mathrm{~km})$ \\
eccentricity $(e)$ & 0.001313349 \\
inclination $(i)$ & $98.000640878^{\circ}$ \\
right ascension & $71.497449685^{\circ}$ \\
of ascending node, RAAN $(\Omega)$ & $69.532933059^{\circ}$ \\
argument of perigee $(\omega)$ & $290.42758119^{\circ}$ \\
mean anomaly $(M)$ & $-69.71353401^{\circ}$ \\
true anomaly $(v)$ & $5834.8051948(\mathrm{~s})$ \\
nodal period $(P)$ &
\end{tabular}

\subsection{Design of Orbital Parameters}

Many publications use the assumption of Keplerian motion for designing the orbital parameters. This, however, causes the mismatch on the ground track. The drift on the ground track also grows over time in the order of several kilometers within a day. The effects of Keplerian assumption would be discussed again at the section of results. Due to inaccuracy of Keplerian motion, we present an iterative methodology for determining the orbital parameters. The technique begins with the preliminary determination.

\subsubsection{Preliminary Analysis}

In order to design the orbit for the SSO, we can start from at determining the semi-major axis from the nodal period in Equation (12) by using approximated Keplerian motion as follows

$$
a=\sqrt[3]{\mu\left(\frac{P}{2 \pi}\right)^{2}}
$$

where $\mu$ is Earth's gravitational constant which is $398,600.4418 \mathrm{~km}^{3} / \mathrm{s}^{2}$ according to WGS84 standard. Now, we determine the inclination angle from the following equation,

$$
i=\cos ^{-1}\left(\frac{-2 \sqrt{a^{7}} \Omega_{\operatorname{Sun}}\left(1-e^{2}\right)^{2}}{3 R_{E}^{2} J_{2} \sqrt{\mu}}\right),
$$

where $J_{2}$ is Earth's zonal harmonic which is $1.082626174 \times 10^{-3}$ according to EGM2008 standard, $\Omega_{\text {Sun }}$ is angular rate of line of node which is $1.991063853 \times 10^{-7} \mathrm{rad} / \mathrm{s}$. Note that we initially assume $e \approx 0.00105$. 
Now we can determine the frozen eccentricity by enforcing the variations of eccentricity and argument of perigee due to $J_{2}$ and $J_{3}$ zonal harmonics to be zero by selecting as follows.

$$
\begin{aligned}
\omega & =90^{\circ} \\
e & \approx-\frac{1}{2} \frac{J_{3} R_{E}}{J_{2} a} \sin i
\end{aligned}
$$

where $J_{3}$ is $-2.532410519 \times 10^{-6}$ according to EGM2008 standard.

Then, we determine the last orbital parameter of right ascension of ascending node (RAAN). The value of RAAN depends on the local solar time and the time. We use the time for computing the angle between prime meridian and vernal equinox $(\theta)$ by following relationships

$$
\begin{aligned}
\theta & =67310.54841+3.164400184812866 \times 10^{9} T_{u t}+0.093104 T_{u t}^{2}-6.2 \times 10^{-6} T_{u t}^{3}, \\
T_{u t} & =\frac{J D_{u t}-2451545.0}{36525}
\end{aligned}
$$

where angle $\theta$ is in the unit of second-angle which equals to $1 / 240^{\circ}, J D_{u t}$ is Julian date with respect to universal time 1 (UT1). One may require $\triangle U T 1$ for the conversion from popular Coordinated Universal Time (UTC) to UT1, however, the error in RAAN angle due to the conversion is relative small in order of $4 \times 10^{-3}$ degree. By determining the angle $\theta$, we can obtain the RAAN of the first orbit on the longitude of zero at a specific time. It is worth noting that the resulting angle of Equation (8) usually requires a modulation of 86,400 for being in the proper range.

\subsubsection{Iterative Method}

Now, we have the orbital parameters from the preliminary determination in last section. Then, the parameters are refined by eliminating the assumption of Keplerian motion. Particularly, the assumption is eliminated by applying analytical relationships of nodal period in Ref. [5], frozen eccentricity in Ref. [6], and inclination in Equation (5). These relationships are complex and coupling together among $a, e$ and $i$. So we cannot analytically solve these three equations to obtain the exact solution in one shot. However, the complexities can be circumvented by applying iterative method which is presented here.

For the concept of iterative method, it contains main 3 steps for solving $a, i$ and $e$ respectively. These main steps sequentially compute and update the values of $a, i$, and $e$ respectively until the changes of all three parameters are within some limits. In the steps of solving $a$ and $e$, the iterative method of root finding, Newton-Raphson, is used.

Let us examine into the detail of the first main step, semi-major axis solver. We can determine the osculating semi-major axis at the equator by solving the following equation [5] for $a$.

$$
P=f(a)=\frac{2 \pi}{\sqrt{\mu}}\left[\sqrt{a^{3}}-\frac{3 J_{2}\left(4-5 \sin ^{2} i\right) R_{E}^{2}}{4 \sqrt{a\left(1-e^{2}\right)}(1+e \cos \omega)^{2}}-\frac{3 J_{2}(1+e \cos \omega)^{3} R_{E}^{2}}{2 \sqrt{a}\left(1-e^{2}\right)^{3}}\right]
$$

Equation (10) can be solved iteratively by applying Newton-Raphson method as follows

$$
a_{i+1}=a_{i}-\frac{f\left(a_{i}\right)-P}{f^{\prime}\left(a_{i}\right)}
$$

where $a_{0}$ can be initially chosen from Keplerian motion as shown in Equation (4), $P$ is desired nodal period from Equation (3), the function $f(a)$ is at Equation (10), and $f^{\prime}(a)$ is

$$
f^{\prime}(a)=\frac{\partial f(a)}{\partial a}=\frac{2 \pi}{\sqrt{\mu}}\left[\frac{3}{2} \sqrt{a}+\frac{3 J_{2}\left(4-5 \sin ^{2} i\right) R_{E}^{2}}{8 \sqrt{a^{3}\left(1-e^{2}\right)}(1+e \cos \omega)^{2}}+\frac{3 J_{2}(1+e \cos \omega)^{3} R_{E}^{2}}{4 \sqrt{a^{3}}\left(1-e^{2}\right)^{3}}\right] .
$$


Noting that the relationship in Equation (10) is also depended on inclination angle $i$ and frozen eccentricity $e$ as they are coupling together.

Next, the second step of inclination solver is straight-forward. The inclination angle is directly obtained from Equation (5) with the parameter $a$ from the first step.

For the third step, the value of frozen eccentricity is determined by solving relationship of

$$
0=g(e)=1+\frac{J_{3} R_{E}\left(\sin ^{2} i-e^{2} \cos ^{2} i\right) \sin \omega}{2 J_{2} a\left(1-e^{2}\right) e \sin i}
$$

with the iterative Newton-Raphson method of

$$
e_{i+1}=e_{i}-\frac{g(e)}{g^{\prime}(e)}
$$

where

$$
g^{\prime}(e)=\frac{\partial g(e)}{\partial e}=\frac{J_{3} R_{E} \sin \omega}{2 J_{2} a \sin i}\left(\frac{4 e^{2} \sin ^{2} i-\sin ^{2} i-e^{4} \cos ^{2} i-e^{2}}{e^{6}-2 e^{4}+e^{2}}\right) .
$$

Noting that the values of $a$ and $i$ using in Equations (13)-(15) are solutions from the previous steps. Then, the computation goes back to the first step and iterates these three steps until the variations of $a$, $i$ and $e$ are substantially small.

\section{Results and Discussion}

We uses the described methodology in previous section to analyze two scenarios:

1. Scenario of TSC satellite flying along the same ground track as THEOS2 main satellite and has a prior local solar of $30 \mathrm{~min}$.

2. Scenario of TSC satellite having 5 times faster revisiting time than THEOS2 main satellite.

\subsection{Scenario 1}

This scenario demonstrates the situation that TSC satellite has the prior local solar time of $30 \mathrm{~min}$. The main reason of scenario is to avoid the congestion of passing satellite at a certain time by assigning an earlier passing time of 9.30 a.m. to TSC satellite. The orbit design also has a consideration of alleviating the adversely long revisiting period of THEOS2 main satellite. The TSC satellite is supposed to be at the beginning of the ground track cycle once the THEOS2 reaches at the point of half cycle.

For this case, the TSC satellite has the same orbit as the reference of $26 D 385 R$. The ground track also appears to be identical as in Figure 1. The resulting orbital parameters are listed in Table 2. There are some errors between the reference orbit in Table 1 and given results because of the differences in analytical expressions and Earth's gravitational model. However, these errors are small and in an order of few meters for semi-major axis. The resulting time of epoch is written in UT1 for ease of computation and it is always within a range of $\pm 0.9 \mathrm{~s}$ from UTC. The resulting date of 9 January 2025 is 1469 days after the reference epoch. This duration corresponds to 56 and $1 / 2$ ground track cycles. In an addition, the altitude at equator is $630.33 \mathrm{~km}$.

Now, let us discuss about the assumption of Keplerian motion in Equation (4) that results the semi-major axis of $7005.0341 \mathrm{~km}$. By applying this value into the Equation (10) with the other parameters from Table 2, it yields the nodal period of $5830.5058240 \mathrm{~s}$. The difference of $4 \mathrm{~s}$ for every revolution means that the ground track error at the equator rapidly increases $1.85 \mathrm{~km}$ for each revolution or $27.47 \mathrm{~km}$ a day. Within 5 days, the error will shifts the ground track from one line to the adjacent line and degrades the accuracy of mission planning. This is the reason why an accurate model for determining semi-major axis and nodal period is required instead of Keplerian assumption. 
Table 2. Results of osculating orbital parameters for both scenarios.

\begin{tabular}{ccc}
\hline & Scenario 1 & Scenario 2 \\
Epoch Time & 9 January 2025 21:30:00.000 UT1 & 1 January 2025 21:30:00.000 UT1 \\
\hline semi-major axis $(a)$ & $7008.474(\mathrm{~km})$ & $6827.726(\mathrm{~km})$ \\
eccentricity $(e)$ & 0.001054258 & 0.001083911 \\
inclination $(i)$ & $97.90756^{\circ}$ & $97.21264^{\circ}$ \\
right ascension & $72.166519^{\circ}$ & $64.281340^{\circ}$ \\
of ascending node, RAAN $(\Omega)$ & $90.00^{\circ}$ & $90.00^{\circ}$ \\
argument of perigee $(\omega)$ & - & - \\
mean anomaly $(M)$ & $270.00^{\circ}$ & $270.00^{\circ}$ \\
true anomaly $(v)$ & $5834.80519(\mathrm{~s})$ & $5610.38961(\mathrm{~s})$ \\
nodal period $(P)$ & &
\end{tabular}

\subsection{Scenario 2}

For the second scenario, we observes the possibility of using TSC satellite as a frequently imaging satellite. For achieving the target, we aim at least 5 time shorter of revisiting time than THEOS2 main satellite. The THEOS2 main satellite has 385 revolutions during 26 days. Then, this scenario has 77 revolutions which is 5 times lesser number of orbits. By this number of orbits, the ground track grid matches of THEOS2 with 5 times larger span as shown in Figure 2. The only possible mean motion is $15+2 / 5$ orbits per day, it is the orbit of $5 D 77 R$. The orbit parameters are shown in Table 2 . Additionally, the altitude at equator is $449.58 \mathrm{~km}$.

The wider width of adjacent ground nodes is promising because the swath width of TSC payload could be larger than THEOS2 main satellite. It possibly cover all areas without unnecessarily small grid. However, due to the lower altitude of the orbit, the spacecraft has higher fuel requirement for station-keeping maneuvering. Therefore, this mission scheme has to be further analyzed.

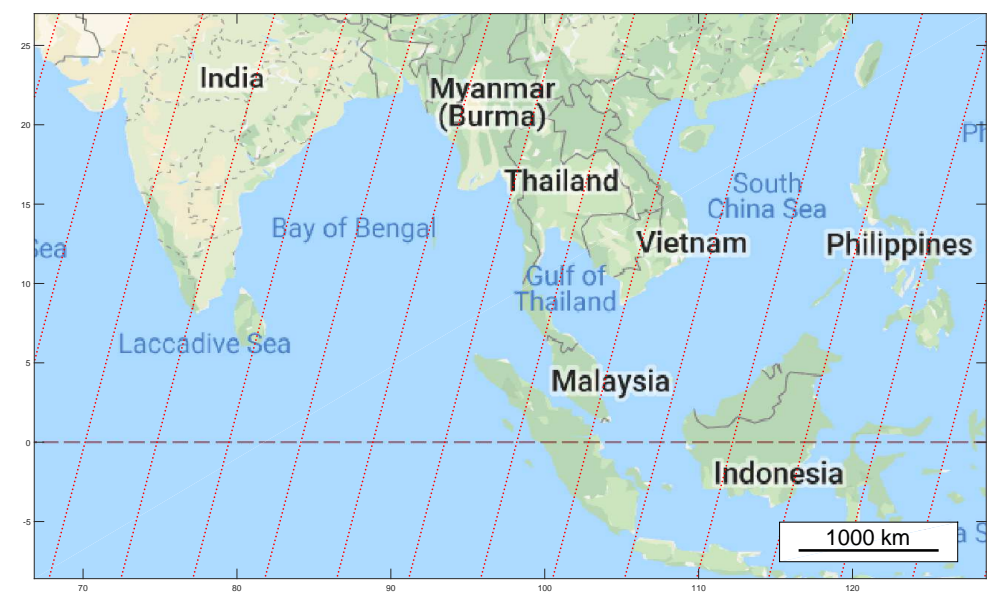

Figure 2. The estimated descending ground track of scenario 2.

\section{Conclusions}

The process of orbit design is iterative. The designed orbits in this paper are required to be reconsidered when more detail about payloads is available or new mission requirement is specified. However, this paper layouts the basic methodology for orbit design and also provides a discussion about the necessity of an accurate orbital period instead of traditional Keplerian orbital period. The proposed orbits are based on the idea of flying as constellation with THEOS2 main satellite. However, this currently is not the mission requirement. There are also some considerations needed to be 
addressed for example the fuel expenditure or $\Delta V$ requirement throughout the mission lifetime or eclipsing time.

Author Contributions: Writing-original draft preparation and methodology, S.S.; data curation, S.J.; review and editing, S.C. All authors have read and agreed to the published version of the manuscript.

Funding: This research was funded by Ministry of Higher Education, Science, Research and Innovation of Thailand.

Acknowledgments: We would like to thank members of fight dynamic team at GISTDA for the data preparation. We also thank Zohar Bar-Yehuda for providing a handy library for earth map figure which is accessible from https://www.github.com/zoharby/plot_google_map.

Conflicts of Interest: The authors declare no conflict of interest.

\section{References}

1. Boain, R.J. A-B-Cs of sun-synchronous orbit mission design. In Proceedings of the 14th AAS/AIAA Space Flight Mechanics Conference, Maui, HI, USA, 8-12 February 2004.

2. Vallado, D.A. Mission Analysis. In Fundamentals of Astrodynamics and Applications; Microcosm Press: Cleveland, OH, USA, 2013; Chapter 11, pp. 837-939.

3. Ravanbakhsh, A.; Franchini, S. System engineering approach to initial design of LEO remote sensing missions. In Proceedings of the 2013 6th International Conference on Recent Advances in Space Technologies (RAST), Istanbul, Turkey, 12-14 June 2013; pp. 659-664.

4. Peter Fortescue, G.S.; Stark, J. Mission Analysis. In Spacecraft Systems Engineering; A John Wiley \& Sons Ltd.: Hoboken, NJ, USA, 2011; Chapter 5, pp. 111-147.

5. Blitzer, L. Nodal period of an earth satellite. AIAA J. 1964, 2, 1459-1460, doi:10.2514/3.2579.

6. Cutting, E.; Frautnick, J.; Born, G. Orbit analysis for SEASAT-A. J. Astronaut. Sci. 1978, 26, 315-342.

(c) 2019 by the authors. Licensee MDPI, Basel, Switzerland. This article is an open access article distributed under the terms and conditions of the Creative Commons Attribution (CC BY) license (http://creativecommons.org/licenses/by/4.0/). 